\title{
Furry is a component of the CCM3-GCKIII signaling pathway
}

\author{
Emmanuel Antwi-Adjei', Alondra S. Burguete ${ }^{2}$, Amin S. Ghabrial ${ }^{1}$ \\ 1Department of Pathology and Cell Biology, Columbia University Irving Medical Center, New York, NY 10032, USA. \\ ${ }^{2}$ Department of Neurology Columbia University Irving Medical Center, New York, NY 10032, USA.
}

Correspondence to: Prof. Amin S. Ghabrial, Department of Pathology and Cell biology, Columbia University Irving Medical Center, 630 W. 168th Street, VP\&S 14-401L, New York, NY 10032, USA. E-mail: asg2236@cumc.columbia.edu

How to cite this article: Antwi-Adjei E, Burguete AS, Ghabrial AS. Furry is a component of the CCM3-GCKIII signaling pathway. Vessel Plus 2021;5:35. https://dx.doi.org/10.20517/2574-1209.2021.48

Received: 23 Mar 2021 First Decision: 15 Apr 2021 Revised: 30 Apr 2021 Accepted: 26 May 2021 First online: 26 May 2021

Academic Editor: Jun Zhang Copy Editor: Yue-Yue Zhang Production Editor: Yue-Yue Zhang

\begin{abstract}
Aim: Mutations in 3 genes encoding proteins of the Cerebral Cavernous Malformations (CCM) ternary complex cause autosomal dominant cerebral vascular disease. Targets of CCM complex regulation have been identified; however, the molecular mechanisms connecting $\mathrm{CCM} 3$ to these downstream effectors remain elusive. We aim to determine the mechanism of CCM3 action by using a Drosophila model to elucidate the signaling pathway downstream of CCM3. Previously, we showed that CCM3 and its binding partner, Germinal Center Kinase 3, are required in tracheal terminal cells to prevent tube morphogenesis defects. Further, we established that GCKIII phosphorylates and directly activates a downstream kinase, Tricornered (Drosophila STK38/38L ortholog). Here we aim to test whether Tricornered-associated scaffolding protein, Furry, is required for CCM3-GCKIII signaling.
\end{abstract}

Methods: We utilized the FRT-FLP system to generate genetic mosaic Drosophila larvae and adults. Mitotic recombination was induced in embryos (trachea) or larvae (wing disc). The animals were heterozygous for the gene of interest (ccm3 or furry), but after recombination, homozygous mutant daughter cells were produced. In addition, the GAL4-UAS system was used to express dominant negative GCKIII in wing disc cells. Mutant cells were analyzed by brightfield and/or fluorescent microscopy.

Results: We find that wing cells mutant for ccm3, or expressing dominant negative GCKIII, produce wing hair defects characteristic of mutations in tricornered and furry. Likewise, tracheal terminal cells mutant for furry produce tube dilation defects characteristic of cells mutant for ccm3 or GCKIII. 
Conclusion: CCM3 and GCKIII act upstream of Furry-Tricornered, suggesting the conservation from yeast of a Hippo-like signaling pathway that regulates morphogenesis. We speculate that some combination of Furry/Furrylike and STK38/38L are therefore likely to act downstream of CCM3 in endothelial cells.

Keywords: CCM3, GCKIII, NDR, STK38/38L, tube morphogenesis, planar cell polarity

\section{INTRODUCTION}

Cerebral cavernous malformations (CCMs) are common vascular defects found in the capillaries of the central nervous system (reviewed in Lampugnani et al. ${ }^{[1]}$ ). Familial CCM shows an autosomal dominant manner of inheritance, with variable expression and incomplete penetrance (reviewed in Riolo et al..$^{[2]}$ ). Somatic loss of a second (Knudsonian 2 hit mechanism) CCM allele ${ }^{[3]}$ is believed to result in localized vascular lesions in which grossly dilated, thin-walled capillaries lacking surrounding support cells undergo repeated hemorrhage leading to headaches, neurological deficits and stroke ${ }^{[4]}$. Lesions are believed to be largely clonally derived, containing few wild type endothelial cells ${ }^{[5]}$ (although also see ${ }^{[6]}$ ). Mutations in three different genes cause disease and account for at least $80 \%$ of cases of familial CCM. These genes - Krev interaction trapped protein 1, Malcavernin, and Programmed Cell Death 10 - are also known as CCM1, $\mathrm{CCM} 2$ and $\mathrm{CCM} 3$, respectively. Generally, the CCM proteins have been thought to act as scaffolding for regulators of cytoskeletal remodeling and/or signaling pathways. The CCM proteins can physically interact, forming a ternary complex bridged by CCM2. The complex may be recruited to the plasma membrane through interaction with a putative transmembrane receptor protein, Heart of Glass ${ }^{[7]}$, or by interaction with the Vascular endothelial growth factor receptor $2^{[8,9]}$. The precise functions of the individual proteins, and of the various complexes, remain key subjects of inquiry. The role of CCM3 has been particularly controversial, as CCM3 is also found in other complexes ${ }^{[10]}$ and has been suggested to operate in a CCM1and CCM2-independent manner ${ }^{[1-115]}$.

Consistent with the idea that CCM3 may act independently of CCM1 and CCM2, mutations in CCM3 cause an earlier lethality in mice, and in humans, patients with CCM3 mutations show a much earlier age of onset and a more severe course of disease $\mathrm{e}^{[12,16-18]}$. Likewise, patients with mutations in CCM3, but not CCM1 or $C C M 2$, develop multiple meningiomas at a high frequency ${ }^{[17]}$. These differences may reflect a requirement for $\mathrm{CCM}_{3}$ in epithelia ${ }^{[19]}$, or may hint at a distinct molecular function for CCM3 even in endothelial cells.

We have turned to the simple Drosophila melanogaster model system to dissect CCM3 function. Importantly, CCM3 is well conserved, but neither CCM1 nor CCM2 orthologs have been reported in flies. In prior work, we identified a requirement for CCM3 and GCKIII in tracheal terminal cell tube morphogenesis ${ }^{[20]}$, and have since gone on to show that GCKIII directly activates the Nuclear Dbf2-related (NDR) kinase, Tricornered (NDR1, 2 in vertebrates, also known as STK38 and STK38L) by phosphorylation $^{[21]}$. Altogether, our work shows that CCM3 is a novel component of an ancient kinase cascade conserved from yeast: the RAM (Regulation of Ace2 and Morphogenesis) pathway in budding yeast, and the MOR (Morphogenesis Orb6 network) pathway in Schizosacharomyces pombe ${ }^{[22]}$.

In yeast and flies, NDR kinase signaling typically requires co-factors including Mob (mps one binder) and Furry family members ${ }^{[23]}$. Four Mob family members have been identified (Mats, Mob2, Mob3 and Mob4) in flies, and single Furry protein (Тao3p in budding yeast and Mor2p in fission yeast). So far, work on the invertebrate fry genes indicates essential roles in Trc signaling, with known requirements in the generation of actin-based cellular protrusions (wing hairs and bristles ${ }^{[24-30]}$ ), in the control of dendritric branching and tiling in dendritic arborization neurons ${ }^{[31-33]}$, and in follicle cell polarity ${ }^{[34]}$. The vertebrate orthologs of Fry 
(FRY and FRYL) have been implicated in several processes ${ }^{[23]}$, including tubulogenesis in the kidney ${ }^{[35,36]}$, but have not yet been linked to vascular biology.

\section{METHODS}

Wings. Young adult flies (newly eclosed up to 1 day old) were anesthetized on $\mathrm{CO}_{2}$ pads and then transferred into $100 \%$ ethanol in glass dissection dishes. Using microdissection spring scissors and Dumont forceps (Fine Science Tools, Foster, CA, USA), the wings were clipped off at their attachment site. Wings were transferred to a drop of Euparal (BioQuip, 6372A), and a coverslip was applied. Weights were placed on the coverslip to flatten the specimens, and slides were allowed to dry at room temperature for 1 day or more before imaging. Images were captured at the wing margin and just distal to the cross-vein connecting wing vein 3 and 4 . For $c c m 3$ mosaic wings, virgin $\mathrm{FRT}^{82 \mathrm{~B}} \mathrm{ccm} 3 / \mathrm{TM} 6 \mathrm{~B}$ flies were crossed with $y, w$, hsFLP ${ }^{122}$; $\mathrm{FRT}^{82 \mathrm{~B}}$ males, and the progeny (embryos and larvae) were subjected to heat shocks $\left(38.5^{\circ} \mathrm{C}\right)$ for $1-2 \mathrm{~h}$ starting at $6 \mathrm{~h}$ after egg lay (a.e.l.). Heat shocks were repeated every day through the end of the $3 \mathrm{rd}$ larval instar. Heat shock induction of Flipase (FLP), a site-specific recombinase, resulted in mitotic recombination between the homologous chromosomes at a centromere proximal FLP Recombinase Target (FRT) site $\left(\mathrm{FRT}^{\mathrm{82} \mathrm{B}}\right)$. As a consequence, genetic mosaic animals were produced with most cells being heterozygous, but with clones of homozyougs wild type and homozygous mutant cells. Mosaic adults were easily recognized by eye color mosaicism, as well as by an unevenness of the wing surfaces (mosaic wings appeared somewhat crinkled rather than the wild type flat appearance that characterized the wings of their TM6B siblings). For GckIII $_{\mathrm{T}_{167 \mathrm{~A}}}$ wing analysis, the wing specific nubGAL4 driver flies were crossed to the UAS-GckIII ${ }_{\mathrm{T} 167 \mathrm{~A}}^{\mathrm{F}_{2}} \mathrm{flies}$ (dominant negative, non-phosphorylatable GCKIII ${ }^{[21]}$ ).

Mosaic analysis of larval trachea. To test the cell autonomous requirement for furry and Mo25 in tracheal cells we generated genetic mosaic animals using FLP-FRT approaches ${ }^{[37,38]}$. The alleles $f r y^{031}$, fr $y^{041}$ and $f r y^{098}$ were gifts from Dr. Sally Horne-Badovinac and encode truncated Fry proteins (Q1008term, W394term and G666term, respectively). The fry $y^{1}$ allele ${ }^{[25]}$ carries a 1 bp deletion causing a frame-shift after aa 403 . Lastly, the $f r y^{s 308}$ allele comes from an EMS/X-ray screen for modifiers of sinaGMR.PN eye phenotype ${ }^{[39]}$. Except for $f r y^{5308}$, all fry alleles were induced on chromosomes carrying FRT ${ }^{80 B}$. For these alleles, mosaic larvae were generated using the MARCM strategy. For $f r y^{5308}$, we generated recombinant chromosomes carrying both the fry mutation and $\mathrm{FRT}^{2 \mathrm{~A}}$, and generated mosaic larvae using a MARCM-related approach we had previously developed, substituting a GFP RNAi transgene for the Tubulin-GAL80 transgene. For Mo25, the D8-2 allele was used ${ }^{[40]}$. Except for analysis of $f r y^{5308}$, virgins of the genotype $y, w, h s F L P 122 ; b t l$-GAL4, UASGFP, UAS-DsRED2nls; Tub-GAL80 FRT80B were crossed to males carrying fry mutant alleles on FRT80B chromosomes in trans to TM3Sb, Twist $>$ GFP. For the fry ${ }^{r 308}$ analysis, virgins of the genotype $y, w$, hsFLP122; btlGAL4, UAS-GFP, UAS-DsRED2nls; UAS-GFP RNAi FRT2A were crossed to males of the genotype fry $y^{308}$ FRT2A/TM3, Sb. Crosses were established with 40 virgins and 20-40 males. After $4 \mathrm{~h}$ at $25^{\circ} \mathrm{C}$, adults were transferred to fresh vials, and the 0-4 h a.e.l. embryos were subjected to 45-60 min heat shock. The embryos were then cultured for an additional 5 days at $25^{\circ} \mathrm{C}$ and analyzed at the third larval instar. Mosaic larvae were identified based on the presence of individual GFP expressing cells in the tracheal system (marking the homozygous mutant clones). The mosaic larvae were then heat killed $\left(\sim 10 \mathrm{~s}\right.$ at $\left.70{ }^{\circ} \mathrm{C}\right)$ in a drop of $50 \%$ glycerol on a slide; a cover slip was added and fluorescent microscopy was used to identify mosaic animals. Mutant terminal cells were analyzed by brightfield microscopy to score the presence and shape of gas-filled tubes. Cells were also analyzed by fluorescent microscopy, with the black space in GFP labeled cells revealing tube lumens, including the lumens of tubes that were not gas-filled (and thus not detectable by brightfield microscopy). Larvae were examined using $20 \times$ and $40 \times$ objectives on a Leica compound fluorescent microscope. Z-stacks were captured for each GFP positive terminal cell using Leica software. Unidentified images were then scored independently for the presence of transition zone tube dilations and 
other defects. In some instances, the position of the terminal cell within the specimen did not allow analysis, and these cells were excluded from counts.

Microscopy. Larvae were imaged using direct fluorescence and Brightfield optics using a Leica DM5500 B upright widefield epifluorescence microscope (Leica Microsystems). Images were acquired using a Leica DFC360FX camera. Z-stacks were captured and processed by deconvolution using Leica Advanced Fluorescence Application Suite (Leica Microsystems). For wing hairs, wings mounted in Euparal were examined with brightfield optics using a Leica DM6000 inverted microscope, and images were captured using a Hammamatsu Orca-R2 Digital CCD camera (C10600, Hamamatsu Photonics).

\section{RESULTS}

We previously identified a requirement for the CCM3-GCKIII signaling pathway in the Drosophila tracheal system $^{[20]}$. Importantly, the CCM3-GCKIII tube morphogenesis program shares common components with an orthologous pathway in the human vascular system (reviewed in Riolo et al..$^{[2]}$ ), which is partially conserved from yeast [Figure 1]. Prior to our work, Tricornered $(\operatorname{Trc})$, the most downstream kinase in the cascade, was best known for its role in the morphogenesis of other tissues ${ }^{[2,3,1,41]}$. We decided to test whether regulation of Trc by CCM3-GCKIII was tissue-specific, or instead might be a general feature of Trc regulation. To do so, we turned to the Drosophila wing, a system widely used for studying developmental signaling pathways and planar cell polarity ${ }^{[22-47]}$.

\section{CCM3-GCKIII pathway regulates wing hair morphogenesis}

In flies, wing epithelia possess planar polarity that is easily read out in each cell by the position and orientation of actin-based cellular protrusions called wing hairs. In wild type wing epithelial cells, a single hair that tapers to a point extends from the posterior vertex of the cell, points distally, and is aligned with the hairs of neighboring cells [Figure 2A]. In tricornered and furry mutant cells, the organization of the actin-based hairs appears to be disrupted such that the hairs split, giving rise to multiple hairs [Figure $2 \mathrm{~B}$ and E] and/or hairs with split ends ${ }^{[24,28]}$. Although prior studies suggest no role for Mo25 in wing hair morphogenesis ${ }^{[30]}$, we decided to determine if disruption of $c \mathrm{~cm} 3$ and GckIII activity would give rise to wing hair defects characteristic of $t r c-f r y^{[26,2]]}$, and we examined wings expressing a dominant negative GCKIII isoform (Figure $2 \mathrm{C}$ and $\mathrm{F}, \mathrm{Gck}_{\mathrm{T} 167 \mathrm{~A}}{ }^{[21]}$ ), as well as $\mathrm{ccm} 3$ mosaic wings [Figure $2 \mathrm{G}$ ]. In both cases, we found that the affected wing cells developed multiple wing hairs with disruption of hair orientation.

\section{Furry is required in tracheal terminal cell tube morphogenesis}

Furry has been identified genetically and biochemically as a partner for $\operatorname{Trc}^{[24,26,27]}$, and has been shown to function together with $\operatorname{Trc}$ in the shaping of actin-based cellular projections (wing hairs, etc.) in epithelial cells, as well as the polarized deposition of basement membrane ${ }^{[23]}$. In neurons, Fry has been shown to function with $\operatorname{Trc}$ in the morphogenesis of dendrites ${ }^{[31]}$. A role for Fry in tubulogenesis has not been examined, although fryl mutants in mice have been reported to have kidney defects attributed to a role in the regulation of a microRNA ${ }^{[35,36]}$. Because Furry appears to be required in all other contexts where $\operatorname{Trc}$ function has been described as necessary for morphogenesis, we tested if furry was also required in the tracheal system. For this analysis we characterized the tracheal terminal cell phenotype of 5 independent mutant alleles of furry, derived from 3 different genetic screen ${ }^{[25,34,39]}$.

As has been the case for other genes in the pathway (tao, ccm3, GckIII ${ }^{[20,21]}$; Figure 3A and B), and is also true for Mo25 [Figure 3C], zygotic loss of fry appeared to uniquely disrupt tube morphogenesis in terminal cells, but not other tracheal cell subtypes. In homozygous fry terminal cells [Figure 3D], we observed a range of phenotypes, running from cells with tubes indistinguishable from wild type, to cells with multiple transition 


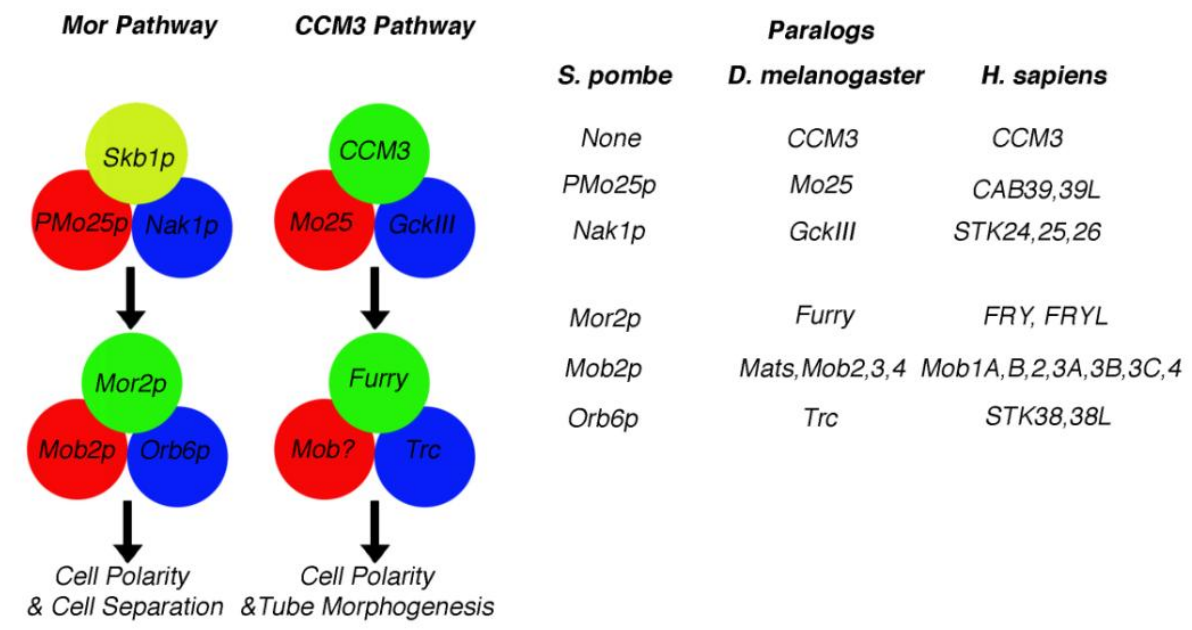

Figure 1. CCM3 is a novel regulator of a conserved Hippo-like signaling pathway. The fission yeast Morphogenesis (Mor) pathway is defined by a short kinase cascade consisting of a Sterile20-like kinase (Nak1p) that phosphorylates and activates an NDR kinase (Orb6p) which in turn phosphorylates effectors required for cell polarity and separation ${ }^{[48]}$. Many of the proteins in this pathway are conserved among eukaryotes, with CCM3 representing a novel scaffolding protein, not present in yeast, for the upstream kinase complex. CCM3 binds directly to GCKIII in human, zebrafish and flies ${ }^{[12,49,50]}$. Likewise, Mo25 can bind directly with Sterile20-like kinases including GCKIII family members ${ }^{[51]}$. Direct interactions between CCM3 and Mo25 have yet to be reported.

zone dilations. The penetrance of the dilation defects varied from allele to allele, with the fry $y^{\text {s30s }}$ allele showing the most penetrant phenotype ( $\sim 80 \%, 25 / 31$ cells showing clear transition zone dilations). In addition to transition zone tube dilations, less prominent tube dilations throughout the terminal cells were also observed, consistent with our prior results for other pathway members. In addition, we observed gasfilling defects and melanization of the transition zone dilation - these defects were less common, with lack of gas-filling observed in about $5 \%$ of mutant cells and melanization in about $2 \%$ of mutant cells $(n=183$ cells scored). All of these defects were also described for tricornered mutant terminal cells ${ }^{[21]}$, and none were observed in wild type control larvae, or in the heterozygous terminal cells in fry mosaic larvae $(n=50$ and 50, respectively). The incomplete penetrance of the fry phenotype may indicate a less stringent requirement for fry than for GckIII and trc, or may reflect the presence of maternally supplied fry mRNA and/or protein.

\section{DISCUSSION}

The molecular mechanisms by which CCM3 acts in the vascular system remain unclear. Strong data support a role of GCKIII family kinases acting together with $\mathrm{CCM} 3$, but the downstream target(s) of the kinase have remained elusive. Our prior work in Drosophila ${ }^{[21]}$ suggests that the NDR kinases (Trc in flies, STK38, STK38L in human) are likely to be the direct targets of the CCM3/GCKIII complex in endothelial cells. Studies linking the scaffolding protein Furry to Trc function implied that Furry was also likely to be required as part of the CCM3-GCKIII signaling cascade. Here we establish that at least in the fly tracheal system, the Furry/Trc complex is regulated by CCM3/GCKIII. The connection between the CCM3-GCKIII complex and a Trc-Fry-Mob complex was suggested by in vitro work using human orthologs ${ }^{[2,53]}$; however, other factors such as mTor or Hippo have also been proposed to act as upstream activators of $\operatorname{Trc}^{[32,33,40,54]}$. Our data in the Drosophila wing demonstrate that at least in two tissues, wing and trachea, Fry/Trc are regulated by the CCM3 pathway. Further studies in additional Trc-requiring tissues, such as neurons and follicle cell epithelia, will need to be carried out determine whether the CCM3 pathway is a tissue-specific or general regulator of Fry/Trc activity. Likewise, it will be critical to extend the analysis to the vertebrate vascular system. If STK38 and STK38L are required downstream of CCM3 in endothelial cells, as expected, 

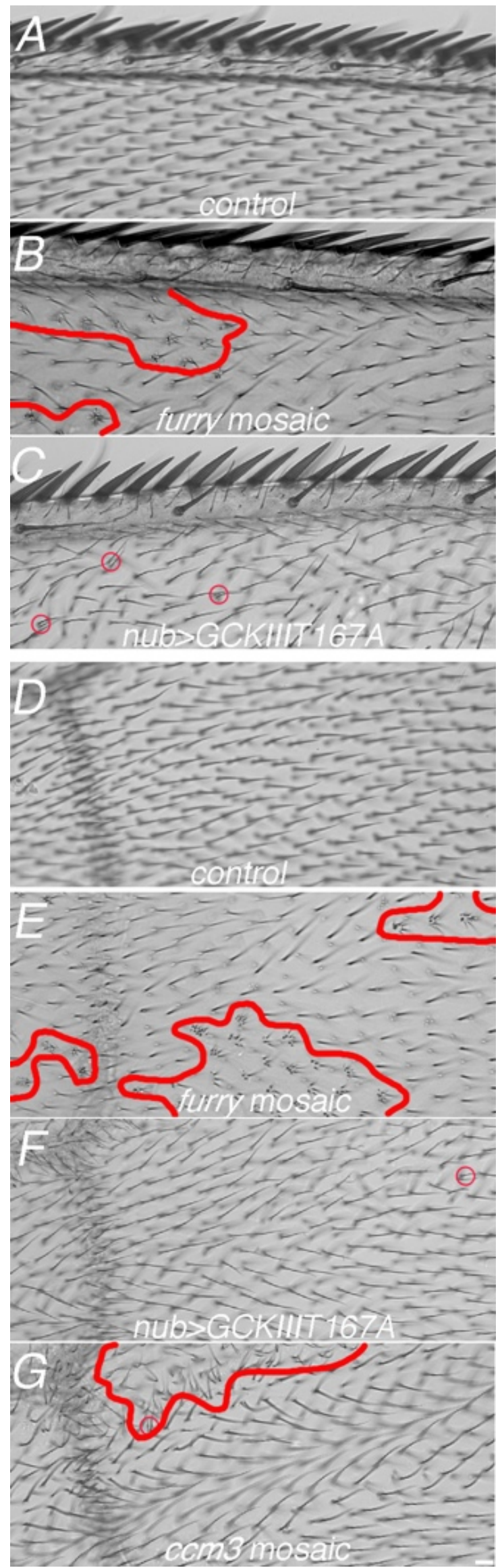
Figure 2. Like mutations in furry, loss of CCM3 or GCKIII function cause wing hair number and polarity defects. (A) Anterior wing margin of a control wild type (OR-R) fly is shown. Note that individual wing hairs are aligned, and point towards the distal wing tip (to the right). In flies mosaic for furry (border between cells in the mutant clone and wild type cells outlined in red) (B), or expressing a dominant negative (non-phophorylatable) GCKIII isoform (GCKIII $\left.\mathrm{T167A}_{\mathrm{A}}\right)(\mathrm{C})$, orientation of the wing hairs is disrupted and (red circles) two or more wing hairs per cell may be produced. (D) Wild type wing hairs in the area immediately proximal to the crossvein connecting veins 3 and 4 (dark pigmentation running vertically on the left of image) are shown. Note the presence of single wing hairs per cell that is oriented towards the distal wing tip (right). In furry clones (E) (clone borders marked in red), or wing cells expressing a dominant negative (non-phophorylatable) GCKIII isoform ( $\mathrm{F}, \mathrm{GCKIII}_{\mathrm{T} 167 \mathrm{~A}}$ ), orientation of the wing hairs is disrupted and (red circles) two or more wing hairs per cell are produced. (G) In wings that are mosaic for loss of ccm3 function (clone outlined in red), mutant cells produce multiple wing hairs and display altered orientation. We note that in addition to the number of wing hairs per cell, mutations in the CCM3/GCKIII-Trc/Fry pathway may also affect planar cell polarity so that the orientation of the hairs appear perturbed. Scale bar in $G$ (for $A-G)=10$ microns.

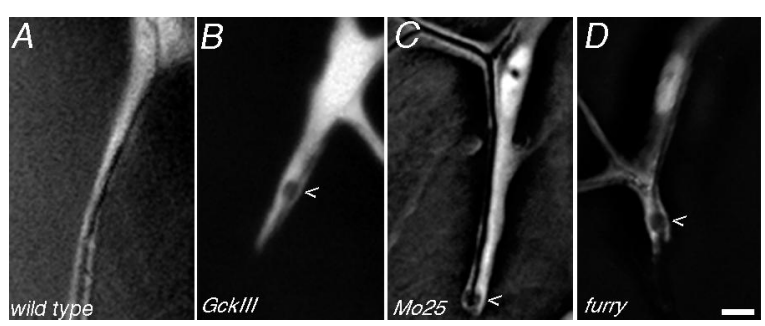

Figure 3. Like mutations in known CCM3-GCKIII signaling pathway components, furry and Mo25 loss of function result in tube dilations in tracheal terminal cells. (A) The most proximal part of a wild type control terminal cell clone (GFP positive labeling of homozygous cells) is shown, depicting the portion of the terminal cell that extends from its intercellular junction with a neighboring stalk cell, at the bottom of the image, to the terminal cell nucleus, at the top of image. In wild type cells the lumen tapers very gradually and evenly from proximal (bottom) to distal (top). (B) In wheezy/Gcklll mutant terminal cells, prominent transition zone (between intercellular junction and terminal cell nucleus) dilations are detected $(<)$. Terminal cells mutant or knocked down for tao, ccm3, trc (not shown, see ${ }^{[20,21]}$ ), (C) Mo25, or (D) furry show identical transition zone dilations. Scale bar $=10$ microns.

it will be important to identify the targets of STK38 and STK38L kinases and to determine if they are universal, differ between epithelial and neural tissues, or show even greater specificity. Given the role for CCM3-GCKIII in Trc-regulated tissue polarity in the wing, it will likewise be of interest to gain mechanistic insight into that process, and to determine whether such functions of CCM3 are related to meningiomas associated with $\mathrm{CCM} 3$ patients. The hypothesis that planar cell polarity (PCP) might be disturbed in meningiomas arises from the observation that a histological characteristic of meningiomas is the whorl formation of neoplastic arachnoid cells. This hypothesis has received some genetic support, as human FAT2, an ortholog of the Drosophila PCP pathway component Fat, has been implicated in meningioma ${ }^{[55]}$. Moreover, Fat protocadherens of the have been implicated in Hippo signaling ${ }^{[5]}$. In the fly, work from Horne-Badovinac and colleagues revealed that Fat2, Fry and $\operatorname{Trc}$ are required to polarize the follicle cell epithelia that surround the Drosophila oocyte. While a role for CCM3-GCKIII in the follicle cells has not yet been described, it is noteworthy that in these cells Trc was found to be distributed in a planar polarized fashion. Strikingly, it was the basolateral aspect of the cells that displayed these asymmetries in $\operatorname{Trc}$ distribution, raising the question of whether the mechanisms involved are related to the canonical PCP pathways, or novel.

\section{DECLARATIONS}

\section{Authors' contributions}

Made substantial contributions to conception and design of the study: Burguete AS, Ghabrial AS Performed data analysis and interpretation: Antwi-Adjei E, Burguete AS, Ghabrial AS 


\section{Availability of data and materials}

All reagents developed in the lab are available upon request.

\section{Financial support and sponsorship}

This work was supported by NIH R01GM089782 to Ghabrial AS.

\section{Conflicts of interest}

All authors declared that there are no conflicts of interest.

\section{Ethical approval and consent to participate}

Not applicable.

\section{Consent for publication}

Not applicable.

\section{Copyright}

(c) The Author(s) 2021.

\section{REFERENCES}

1. Lampugnani MG, Malinverno M, Dejana E, Rudini N. Endothelial cell disease: emerging knowledge from cerebral cavernous malformations. Curr Opin Hematol 2017;24:256-64. DOI PubMed

2. Riolo G, Ricci C, Battistini S. Molecular genetic features of cerebral cavernous malformations (CCM) patients: an overall view from genes to endothelial cells. Cells 2021;10:704. DOI PubMed PMC

3. Pagenstecher A, Stahl S, Sure U, Felbor U. A two-hit mechanism causes cerebral cavernous malformations: complete inactivation of CCM1, CCM2 or CCM3 in affected endothelial cells. Hum Mol Genet 2009;18:911-8. DOI PubMed PMC

4. Haasdijk RA, Cheng C, Maat-Kievit AJ, Duckers HJ. Cerebral cavernous malformations: from molecular pathogenesis to genetic counselling and clinical management. Eur J Hum Genet 2012;20:134-40. DOI PubMed PMC

5. Detter MR, Snellings DA, Marchuk DA. Marchuk. Cerebral cavernous malformations develop through clonal expansion of mutant endothelial cells. Circ Res 2018;123:1143-51. DOI PubMed PMC

6. Malinverno M, Maderna C, Abu Taha A, et al. Endothelial cell clonal expansion in the development of cerebral cavernous malformations. Nat Commun 2019;10:2761. DOI PubMed PMC

7. Kleaveland B, Zheng X, Liu JJ, et al. Regulation of cardiovascular development and integrity by the heart of glass-cerebral cavernous malformation protein pathway. Nat Med 2009;15:169-76. DOI PubMed PMC

8. Kean MJ, Ceccarelli DF, Goudreault M, et al. Structure-function analysis of core STRIPAK Proteins: a signaling complex implicated in Golgi polarization. J Biol Chem 2011;286:25065-75. DOI PubMed PMC

9. Preisinger C, Short B, De Corte V, et al. YSK1 is activated by the Golgi matrix protein GM130 and plays a role in cell migration through its substrate 14-3-3zeta. J Cell Biol 2004;164:1009-20. DOI PubMed PMC

10. Fidalgo M, Fraile M, Pires A, Force T, Pombo C, Zalvide J. CCM3/PDCD10 stabilizes GCKIII proteins to promote Golgi assembly and cell orientation. J Cell Sci 2010;123:1274-84. DOI PubMed

11. Yoruk B, Gillers BS, Chi NC, Scott IC. Ccm3 functions in a manner distinct from Ccm1 and Ccm2 in a zebrafish model of CCM vascular disease. Dev Biol 2012;362:121-31. DOI PubMed

12. Chan AC, Drakos SG, Ruiz OE, et al. Mutations in 2 distinct genetic pathways result in cerebral cavernous malformations in mice. $J$ Clin Invest 2011;121:1871-81. DOI PubMed PMC

13. Zhu Y, Wu Q, Xu JF, et al. Differential angiogenesis function of CCM2 and CCM3 in cerebral cavernous malformations. Neurosurg Focus 2010;29:E1. DOI PubMed

14. Jenny Zhou H, Qin L, Zhang H, et al. Endothelial exocytosis of angiopoietin-2 resulting from CCM3 deficiency contributes to cerebral cavernous malformation. Nat Med 2016;22:1033-42. DOI PubMed PMC

15. Zhang Y, Tang W, Zhang H, et al. A network of interactions enables CCM3 and STK24 to coordinate UNC13D-driven vesicle exocytosis in neutrophils. Dev Cell 2013;27:215-26. DOI PubMed PMC

16. Denier C, Labauge P, Bergametti F, et al. Genotype-phenotype correlations in cerebral cavernous malformations patients. Ann Neurol 2006;60:550-6. DOI PubMed

17. Riant F, Bergametti F, Fournier HD, et al. CCM3 mutations are associated with early-onset cerebral hemorrhage and multiple meningiomas. Mol Syndromol 2013;4:165-72. DOI PubMed PMC

18. Shenkar R, Shi C, Rebeiz T, et al. Exceptional aggressiveness of cerebral cavernous malformation disease associated with PDCD10 mutations. Genet Med 2015;17:188-96. DOI PubMed PMC

19. Tang AT, Sullivan KR, Hong CC, et al. Distinct cellular roles for PDCD10 define a gut-brain axis in cerebral cavernous malformation. Sci Transl Med 2019;11:eaaw3521. DOI PubMed PMC 
20. Song Y, Eng M, Ghabrial AS. Focal defects in single-celled tubes mutant for Cerebral cavernous malformation 3, GCKIII, or NSF2. Dev Cell 2013;25:507-19. DOI PubMed PMC

21. Poon CLC, Liu W, Song Y, et al. A Hippo-like signalling pathway controls tracheal morphogenesis in Drosophila melanogaster. Dev Cell 2018;47:564-75. DOI PubMed PMC

22. Maerz S, Seiler S. Tales of RAM and MOR: NDR kinase signaling in fungal morphogenesis. Curr Opin Microbiol 2010;13:663-71. DOI PubMed

23. Nagai T, Mizuno K. Multifaceted roles of Furry proteins in invertebrates and vertebrates. J Biochem 2014;155:137-46. DOI PubMed

24. He Y, Fang X, Emoto K, Jan YN, Adler PN. The tricornered Ser/Thr protein kinase is regulated by phosphorylation and interacts with furry during Drosophila wing hair development. Mol Biol Cell 2005;16:689-700. DOI PubMed PMC

25. Cong J, Geng W, He B, Liu J, Charlton J, Adler PN. The furry gene of Drosophila is important for maintaining the integrity of cellular extensions during morphogenesis. Development 2001;128:2793-802. PubMed

26. Fang X, Adler PN. Regulation of cell shape, wing hair initiation and the actin cytoskeleton by Trc/Fry and Wts/Mats complexes. Dev Biol 2010;341:360-74. DOI PubMed PMC

27. Fang X, Lu Q, Emoto K, Adler PN. The Drosophila Fry protein interacts with Trc and is highly mobile in vivo. BMC Dev Biol 2010;10:40. DOI PubMed PMC

28. Geng W, He B, Wang M, Adler PN. The tricornered gene, which is required for the integrity of epidermal cell extensions, encodes the Drosophila nuclear DBF2-related kinase. Genetics 2000;156:1817-28. PubMed PMC

29. He B, Adler PN. The genetic control of arista lateral morphogenesis in Drosophila. Dev Genes Evol 2002;212:218-29. DOI PubMed

30. He Y, Emoto K, Fang X, et al. Drosophila Mob family proteins interact with the related tricornered (Trc) and warts (Wts) kinases. Mol Biol Cell 2005;16:4139-52. DOI PubMed PMC

31. Emoto K, He Y, Ye B, et al. Control of dendritic branching and tiling by the Tricornered-kinase/Furry signaling pathway in Drosophila sensory neurons. Cell 2004;119:245-56. DOI PubMed

32. Emoto K, Parrish JZ, Jan LY, Jan YN. The tumour suppressor Hippo acts with the NDR kinases in dendritic tiling and maintenance. Nature 2006;443:210-3. DOI PubMed

33. Koike-Kumagai M, Yasunaga K, Morikawa R, Kanamori T, Emoto K. The target of rapamycin complex 2 controls dendritic tiling of Drosophila sensory neurons through the Tricornered kinase signalling pathway. EMBO J 2009;28:3879-92. DOI PubMed PMC

34. Horne-Badovinac S, Hill J, Gerlach G 2nd, Menegas W, Bilder D. A screen for round egg mutants in Drosophila identifies tricornered, furry, and misshapen as regulators of egg chamber elongation. G3 (Bethesda) 2012;2:371-8. DOI PubMed PMC

35. Byun YS, Kim EK, Araki K, et al. Fryl deficiency is associated with defective kidney development and function in mice. Exp Biol Med (Maywood) 2018;243:408-17. DOI PubMed PMC

36. Espiritu EB, Crunk AE, Bais A, et al. The Lhx1-Ldb1 complex interacts with Furry to regulate microRNA expression during pronephric kidney development. Sci Rep 2018;8:16029. DOI PubMed PMC

37. Lee T, Luo L. Mosaic analysis with a repressible cell marker for studies of gene function in neuronal morphogenesis. Neuron 1999;22:451-61. DOI PubMed

38. Ghabrial AS, Levi BP, Krasnow MA. A systematic screen for tube morphogenesis and branching genes in the Drosophila tracheal system. PLoS Genet 2011;7:e1002087. DOI PubMed PMC

39. Neufeld TP, Tang AH, Rubin GM. Rubin. A genetic screen to identify components of the sina signaling pathway in Drosophila eye development. Genetics 1998;148:277-86. PubMed PMC

40. Yamamoto Y, Izumi Y, Matsuzaki F. The GC kinase Fray and Mo25 regulate Drosophila asymmetric divisions. Biochem Biophys Res Commun 2008;366:212-8. DOI PubMed

41. Natarajan R, Barber K, Buckley A, Cho P, Egbejimi A, Wairkar YP. Tricornered kinase regulates synapse development by regulating the levels of Wiskott-Aldrich syndrome protein. PLoS One 2015;10:e138188. DOI PubMed PMC

42. Peng Y, Axelrod JD. Asymmetric protein localization in planar cell polarity: mechanisms, puzzles, and challenges. Curr Top Dev Biol 2012;101:33-53. DOI PubMed PMC

43. Strutt H, Strutt D. How do the Fat-Dachsous and core planar polarity pathways act together and independently to coordinate polarized cell behaviours? Open Biol 2021;11:200356. DOI PubMed PMC

44. Adler PN. The frizzled/stan pathway and planar cell polarity in the Drosophila wing. Curr Top Dev Biol 2012;101:1-31. DOI PubMed PMC

45. Bier E. Drawing lines in the Drosophila wing: initiation of wing vein development. Curr Opin Genet Dev 2000;10:393-8. DOI PubMed

46. Matsuda S, Harmansa S, Affolter M. BMP morphogen gradients in flies. Cytokine Growth Factor Rev 2016;27:119-27. DOI PubMed

47. Swarup S, Verheyen EM. Wnt/Wingless signaling in Drosophila. Cold Spring Harb Perspect Biol 2012;4:a007930. DOI PubMed PMC

48. Gupta S, McCollum D. Crosstalk between NDR kinase pathways coordinates cell cycle dependent actin rearrangements. Cell Div 2011;6:19. DOI PubMed PMC

49. Voss K, Stahl S, Schleider E, et al. CCM3 interacts with CCM2 indicating common pathogenesis for cerebral cavernous malformations. Neurogenetics 2007;8:249-56. DOI PubMed

50. Voss K, Stahl S, Hogan BM, et al. Functional analyses of human and zebrafish 18-amino acid in-frame deletion pave the way for domain mapping of the cerebral cavernous malformation 3 protein. Hum Mutat 2009;30:1003-11. DOI PubMed

51. Filippi BM, de los Heros P, Mehellou Y, et al. MO25 is a master regulator of SPAK/OSR1 and MST3/MST4/YSK1 protein kinases. EMBO J 2011;30:1730-41. DOI PubMed PMC 
52. Avruch J, Zhou D, Fitamant J, Bardeesy N, Mou F, Barrufet LR. Protein kinases of the Hippo pathway: regulation and substrates. Semin Cell Dev Biol 2012;23:770-84. DOI PubMed PMC

53. Gundogdu R, Hergovich A. MOB (Mps one Binder) proteins in the hippo pathway and cancer. Cells 2019;8:569. DOI PubMed PMC

54. Wu Z, Sawada T, Shiba K, et al. Tricornered/NDR kinase signaling mediates PINK1-directed mitochondrial quality control and tissue maintenance. Genes Dev 2013;27:157-62. DOI PubMed PMC

55. Tate G, Kishimoto K, Mitsuya T. A novel mutation of the FAT2 gene in spinal meningioma. Oncol Lett 2016;12:3393-6. DOI PubMed PMC

56. Peng Z, Gong Y, Liang X. Role of FAT1 in health and disease. Oncol Lett 2021;21:398. DOI PubMed PMC 